\title{
A brief assessment of physical functioning for prostate cancer patients
}

\author{
This article was published in the following Dove Press journal: \\ Patient Related Outcome Measures \\ 17 June 2010 \\ Number of times this article has been viewed
}

\author{
Jin-Shei Lai' \\ Rita Bode ${ }^{2}$ \\ Hwee-Lin Wee ${ }^{3}$ \\ David Eton ${ }^{4}$ \\ David Cella' \\ 'Department of Medical Social \\ Sciences, ${ }^{2}$ Department of Physical \\ Medicine and Rehabilitation, Feinberg \\ School of Medicine, Northwestern \\ University, IL USA; ${ }^{3}$ Department of \\ Pharmacy, National University of \\ Singapore, Singapore; ${ }^{4}$ Division of \\ Health Care Policy and Research, \\ Department of Health Sciences \\ Research, Mayo Clinic, Rochester, MN, \\ USA
}

Correspondence: Jin-Shei Lai Research Associate Professer, Medical Social Sciences and Pediatrics, Feinberg School of Medicine, Northwestern University, 645 North Michigan Ave, Chicago, IL 606 I I, USA

Tel +l 3125031725

$\mathrm{Fax}+\mathrm{I} 3125036743$

Email Js-lai@northwestern.edu

\begin{abstract}
We aimed to validate a more rapid, yet reliable means of assessing physical function (PF) for patients with prostate cancer. The sample included 128 prostate cancer patients recruited from urology and general oncology clinics at two Chicago-area hospitals. The main outcome measures were: A 36-item PF item bank that included a 5-item short form (BriefPF) and the 10-item PF subscale (PF-10) from the Medical Outcomes Study SF-36. Validity, information function, and relative precision (calculated using Rasch analysis and raw scores) of the BriefPF were compared to the PF-10 and the full PF item bank. We found that the BriefPF and PF-10 were strongly correlated $(\mathrm{r}=0.85)$ with the PF bank, and all three scales differentiated patients according to performance status $\left(\mathrm{F}_{\mathrm{PF} \text { bank }}(2,124)=32.51 P<0.001, \mathrm{~F}_{\mathrm{PF}-10}(2,121)=27.35\right.$ $\left.P<0.001, \mathrm{~F}_{\text {BriefPF }}(2,123)=38.40 P<0.001\right)$. BriefPF has excellent precision relative to the PF-10 in measuring patients with different performance status levels. The Rasch-based information function indicated that the BriefPF was more informative than PF-10 in measuring moderate to higher functioning patients. Hence, the BriefPF offers a parsimonious and precise measure of PF for use among men with prostate cancer, and may aid in the timely inclusion of patient-reported outcomes in treatment decision-making.
\end{abstract}

Keywords: quality-of-life, item bank, short-form, Medical Outcomes Study

\section{Introduction}

Prostate cancer is the most commonly diagnosed nonskin cancer, and the second leading cause of cancer death among men in the United States $(9 \%$ of all cancer-related deaths). ${ }^{1}$ While clinical implications vary according to stage and type of treatment, individuals diagnosed with prostate cancer are subject to many complications that affect health-related quality-of-life (HRQL). These complications may be associated with either the disease ${ }^{2-4}$ or treatment ${ }^{5-8}$ and include urinary, bowel, and sexual dysfunction; fatigue; pain; and mental distress. ${ }^{9}$ Numerous studies have also shown general physical deficits and impairments to be common both after and during prostate cancer treatment. ${ }^{6,10-13}$ Deficits in physical functioning (PF) can impair the ability to carry out various activities of daily living, ranging from basic self care to instrumental activities of daily living, to more challenging and vigorous activities. Several studies have also reported that physical performance and falls may occur at higher prevalence in prostate cancer patients, particularly those on androgen deprivation therapy. ${ }^{14,15}$ Additionally, as the majority of prostate patients are elderly (about 63\% of prostate cancers are diagnosed in men 65 years and older), ${ }^{1}$ physical performance which is linked to function is thus of great importance to elderly patients. While most men treated for prostate cancer will experience declines in PF, the deficits can vary across disease stage 
and treatment. ${ }^{10}$ Today, when assessing PF, one can choose from among several questionnaire options, each with very similar item content. The similarity of these questionnaires to one another suggests a common metric could be derived, which can enhance interpretation and communication about differences in PF across individuals or in the same person over time. Such an advance could increase the value of PF assessment in clinical practice. A PF item bank for cancer patients was created to meet such needs. ${ }^{16,17}$ Item banks are a collection of carefully calibrated questions that develop, define, and quantify a common trait and thus provide an operational definition of a given concept. ${ }^{16}$ Item banks allow for greater precision in HRQL assessment that can then be tailored using adaptive testing, which involves the selection of items based on previous patient responses. We have previously developed an abbreviated form of the PF item bank for clinical research in a general cancer patient population (all cancer types excluding prostate cancer: 204; prostate cancer: 198) and plan to test it further among patients with prostate cancer. ${ }^{17}$

In this study, we validated a brief and clinically useful measure of PF derived from the larger PF item bank that can be applied in prostate cancer studies and practice. Prior research has supported the validity of shorter tools for evaluating HRQL outcomes. ${ }^{18}$ We pilot tested the PF items on a sample of men diagnosed with prostate cancer. We compared the performance of the BriefPF with the complete PF item bank, and also with patient responses to the 10-item PF subscale (PF-10) from the SF-36 functional status measure. ${ }^{19}$

\section{Materials and methods}

\section{Sample}

Patients with an existing diagnosis of prostate cancer, regardless of disease stages and types of treatments, were recruited from the urology and general oncology clinics at the Veterans Affairs Chicago Healthcare System-Lakeside Division (Chicago, IL); and North Shore University Health System (formerly Evanston Northwestern Healthcare; Evanston, IL).
The study protocol was approved by Institutional Review Boards at each participating institution.

\section{Instruments and procedure}

Consenting patients provided sociodemographic, treatment, and disease-specific information by interview. PF was assessed using a 36-item "core" PF item bank, ${ }^{20}$ which includes the 5-item shortened assessment form (BriefPF), and the PF-10 from the SF-36. ${ }^{19}$ The item bank included items that represent basic and instrumental activities of daily living. All items were drawn, with permission, from existing HRQL questionnaires, ${ }^{21-26}$ and consisted of different types of rating scales. The process for developing this item bank and the BriefPF, along with their psychometric properties, were reported previously by Bode and colleagues. ${ }^{17,20}$ The BriefPF items cover the range of functional activities of various levels of difficulty (see Table 1), and use a single five-point Likert rating scale whose responses can be summed and converted to a total score (ranging from 21 to 70 ). The PF items were administered to patients using a computer-based testing platform.

\section{Data analysis}

All responses to the PF items were co-calibrated to the item parameter based on a general cancer population. ${ }^{17}$ This allows clinicians to compare PF across different types of cancer. All raw scores were transformed into interval measures via a probabilistic logistic model (ie, Rasch measurement model) to create a common metric on an interval scale, which is generally preferred over ordinal rating scale scores. This co-calibration enables one to "crosswalk" from scores on one instrument to another as long as they are derived from the same item bank, such as the PF-10 and BriefPF. An example crosswalk table can be found in Bode et al. ${ }^{17}$ The coverage of the full spectrum of PF was then compared across the three instruments, with the expectation that the full bank would be the most comprehensive in its coverage. We examined the interrelationship of scores on the full PF

Table I Items and rating scale for the BriefPF

\begin{tabular}{|c|c|c|c|c|c|}
\hline & $\begin{array}{l}\text { None of } \\
\text { the time }\end{array}$ & $\begin{array}{l}\text { A little of } \\
\text { the time }\end{array}$ & $\begin{array}{l}\text { Some of } \\
\text { the time }\end{array}$ & $\begin{array}{l}\text { Most of } \\
\text { the time }\end{array}$ & $\begin{array}{l}\text { All of } \\
\text { the time }\end{array}$ \\
\hline Are you able to move around the house? & I & 2 & 3 & 4 & 5 \\
\hline Are you able to get around to do your daily activities? & I & 2 & 3 & 4 & 5 \\
\hline Are you limited in pursuing your hobbies of other leisure time activities? & 5 & 4 & 3 & 2 & 1 \\
\hline $\begin{array}{l}\text { As a result of your physical health, have you accomplished } \\
\text { less than you would like? }\end{array}$ & 5 & 4 & 3 & 2 & I \\
\hline $\begin{array}{l}\text { As a result of your physical health, have you had difficulty } \\
\text { performing your work or usual daily activities? }\end{array}$ & 5 & 4 & 3 & 2 & I \\
\hline
\end{tabular}


item bank, the BriefPF and the PF-10 and then compared the scale information function of PF-10 and BriefPF across the PF continuum. Next, the ability of each of the three scales to differentiate patients according to performance status was examined. We hypothesized that a PF measure should be sensitive to differences across status levels. We compared the precision of the PF-10 and BriefPF as compared to the full PF item bank using the Eastern Cooperative Oncology Group (ECOG) Performance Status Rating (PSR) as the clinical anchor. ${ }^{27}$ The relative precision (RP) estimates were computed from the ratio pairwise $\mathrm{F}$ statistics using $\mathrm{PF}$ bank as the standard measure, ie, $\mathrm{RP}_{\mathrm{PF}-10}=\mathrm{F}_{\mathrm{PF}-10} / \mathrm{F}_{\mathrm{PF} \text { bank }}$ and $\mathrm{RP}_{\mathrm{BriefPF}}=$ $\mathrm{F}_{\mathrm{BriefPF}} / \mathrm{F}_{\mathrm{PF} \text { bank }}$. RP estimates indicate in proportional terms how much more or less precise a measure is in relation to the standard. ${ }^{28}$ Given the invert reciprocal relationship between information function and error function produced by the Rasch analysis, we also compared measurement precision between PF-10 and BriefPF, by depicting their scale information function curves. A scale with larger information function indicates a more reliable measure. As the information function is a function of the number of items included in the scale, we did not calculate information function for the PF bank because it contains substantially more items than both the PF-10 (10 items) and the BriefPF (five items). WINSTEPS $^{29}$ was used to calibrate items and calculate the information function. SPSS software (SPSS lnc., Chicago, IL) was used to conduct ANOVA and relative precision.

\section{Results}

A total of 128 prostate cancer patients were recruited for the study. The average age was 73.1 years $(\mathrm{SD}=8.7) ; 57.5 \%$ were Caucasian and $41.7 \%$ were African American. Approximately half of participants (46.5\%) had a high school level of education or less, while $22.8 \%$ reported some college, $19.7 \%$ were college graduates, and $11.0 \%$ possessed an advanced degree. A majority were diagnosed with localized disease $(82.8 \%), 14(10.9 \%)$ had metastatic prostate cancer, and the clinical stage for eight patients was unknown.

Rasch measurement scores were computed for the 36 core-items of the PF bank $(\mathrm{M}=1.30, \mathrm{SD}=2.00)$, the PF-10 $(\mathrm{M}=0.31, \mathrm{SD}=2.38)$, and BriefPF $(\mathrm{M}=1.30, \mathrm{SD}=2.00)$. Pearson correlation coefficients were calculated between each of the three PF measures (Table 2). As expected, both the BriefPF and PF-10 were strongly and significantly correlated with the PF bank $(0.85$ and $0.85, P<0.001)$. Correlation between PF-10 and BriefPF was also significantly strong (0.80, $P<0.001)$. The PF bank, PF-10, and BriefPF all were able to differentiate patients according to the ECOG
Table 2 Pearson correlation coefficients between the physical functioning measures (validity analysis)

\begin{tabular}{|c|c|c|c|}
\hline Measure & Item bank ${ }^{\mathbf{a}}$ & PF-IO & BriefPF \\
\hline Item Bank ${ }^{\mathrm{a}}$ & I & $0.853^{* k+k k}$ & $0.850^{* * k+k}$ \\
\hline PF-10 & - & 1 & $0.795^{\text {*ata }}$ \\
\hline BriefPF & - & - & I \\
\hline
\end{tabular}

Notes: altem bank scores were adjusted for overlapping items by deleting PF-10 items (when compared to PF-10) or BriefPF (when compared to BriefPF). ${ }^{*{ }^{*} \mathrm{P}} \mathrm{P}<$ 0.001 .

Abbreviations: PF-10, Physical Functioning-10 items; extracted from the short form 36 items; BriefPF Brief Physical Functioning - 5 items.

PSR levels (scores of 2-4 were combined due to the small sample size in these three categories), $\mathrm{F}_{\mathrm{PF} \text { bank }}(2,124)=32.51$ $P<0.001, \mathrm{~F}_{\mathrm{PF}-10}(2,121)=27.35 P<0.001, \mathrm{~F}_{\text {BriefPF }}(2,123)=$ $38.40 P<0.001$, respectively (shown on Table 3 ). Using the PF bank as the standard, we calculated the relative precision (RP) of PF-10 and BriefPF, in which $\mathrm{RP}_{\mathrm{PF}-10}=0.84$ and $\mathrm{RP}_{\text {BriefPF }}=1.18$. These results suggest, when Rasch measures were used, that the BriefPF is $18 \%$ more precise and PF-10 is $16 \%(1-0.84)$ less precise than the complete PF bank in measuring patients with different ECOG PSR. When raw scores were used, all three measures were still able to differentiate patients according to the ECOG PSR levels, $\mathrm{F}_{\mathrm{PF} \text { bank }}(2,124)=47.52 P<0.001, \mathrm{~F}_{\mathrm{PF}-10}(2,121)=27.74$ $P<0.001, \mathrm{~F}_{\text {BriefPF }}(2,124)=46.68 P<0.001$, respectively. Consequently, the RP of PF-10 and BriefPF was 0.58 and 0.98, respectively, suggesting BriefPF produced comparable precision as the complete PF item bank.

Figure 1 illustrates the information function curves for PF-10 and BriefPF on the PF continuum, as defined by the PF bank. It was noted that the BriefPF had high information function between PF measures -0.6 and 2.8. When matching the PF continuum to the ECOG PSR levels, the PF-10 was more informative in measuring lower functioning patients (ECOG $=2$ to 4 and part of 1 ) while BriefPF was more informative in measuring moderate to higher functioning patients $(\mathrm{ECOG}=0$ and part of 1$)$.

\section{Discussion}

This paper provides evidence of the ability to develop a brief and practical PF short form, targeting on a specific cancer type from a relative large PF item bank that was developed based on the general cancer population. Our results suggest that the carefully developed BriefPF can offer clinicians a parsimonious, yet reliable, PF measure among prostate cancer patients, particularly those who had better functioning (ECOG PSR ratings $=0$ or 1 ). Specifically, the 5-item BriefPF and 
Table 3 Discrimination of three physical functioning measures to patients' self-reported functional performance

3a. Rasch measure-based analysis

\begin{tabular}{|c|c|c|c|}
\hline ECOG PSR levels & $\begin{array}{l}\text { Item bank } F(2, I 24)=32.5 I \\
P<0.00 \text { I }\end{array}$ & $\begin{array}{l}P F=10 F(2,12 I)=27.35 \\
P<0.00 I\end{array}$ & $\begin{array}{l}\text { BriefPF } F(2,123)=38.40 \\
P<0.00 I\end{array}$ \\
\hline $0(n=63)$ & $2.04(1.83)$ & $\mathrm{I} .14(2.2 \mathrm{I})$ & $2.14(1.63)$ \\
\hline I $(n=46)$ & $0.36(1.38)$ & $-0.62(1.70)$ & $0.29(1.54)$ \\
\hline $2-4(n=18)$ & $-1.24(1.63)$ & $-2.80(2.10)$ & $-1.42(1.86)$ \\
\hline Relative precision & 1.00 & $\mathrm{RP}_{\mathrm{PF}-10}=0.84$ & $\mathrm{RP}_{\text {BrieffF }}=1.18$ \\
\hline
\end{tabular}

3b. Raw score-based analysis

\begin{tabular}{|c|c|c|c|}
\hline ECOG PSR levels & $\begin{array}{l}\text { Item bank } F(2,124)=47.5 \text { I } \\
P<0.00 \text { I }\end{array}$ & $\begin{array}{l}P F-10 F(2,12 I)=27.738 \\
P<0.00 I\end{array}$ & $\begin{array}{l}\text { BriefPF F }(2,124)=46.683 \\
P<0.001\end{array}$ \\
\hline $0(n=63)$ & $127.38(19.30)$ & $25.49(4.60)$ & $20.6(2.95)$ \\
\hline$I(n=46)$ & $105.85(21.50)$ & $21.81(4.29)$ & $16.84(3.57)$ \\
\hline $2-4(n=18)$ & $75.23(23.72)$ & I $6.5(4.79)$ & II.44 (4.83) \\
\hline Relative precision & 1.00 & $\mathrm{RP}_{\mathrm{PF}-10(\mathrm{raw})}=0.58$ & $\mathrm{RP}_{\text {BriefPF (raw) }}=0.98$ \\
\hline
\end{tabular}

Note: Higher scores represent better physical function.

Abbreviations: BriefPF, brief physical functioning (5 items); ECOG PSR, eastern cooperative oncology group performance status rating; F, F-statistics; PF-I0, physical functioning ( 10 items; extracted from the short form 36 items); RP, relative precision.

the 10-item PF-10 were correlated to the 36-item PF item bank with a similar strength, and yet the BriefPF demonstrated greater precision for the measurement of PF across ECOG PSR levels regardless of using measures derived from Rasch analysis or raw scores. We were surprised to note that Rasch measure based RP of the BriefPF was greater than 1, indicating the BriefPF exhibited better precision than the PF-item bank. The linear transformation diminishes ceiling and floor effects for both scales. Both the BriefPF and the PF item bank contain items that cover the whole PF continuum. BriefPF contains five items that are equally distributed on the continuum while PF item bank consists of more items; however some bank items have similar locations on the continuum. To evaluate whether the linear transformation of the raw scores contributed to the high RP of the BriefPF, we estimated raw scores based PR. Results showed BriefPF has the same level of the precision as the PF item bank, but the RP of the PF-10 was not as good as that of the BriefPF. We therefore confidently conclude that a carefully developed shortform is able to provide an efficient yet comparable precise PF estimation compared to measures produced by the PF item bank. It should be noted that while our reporting of relative

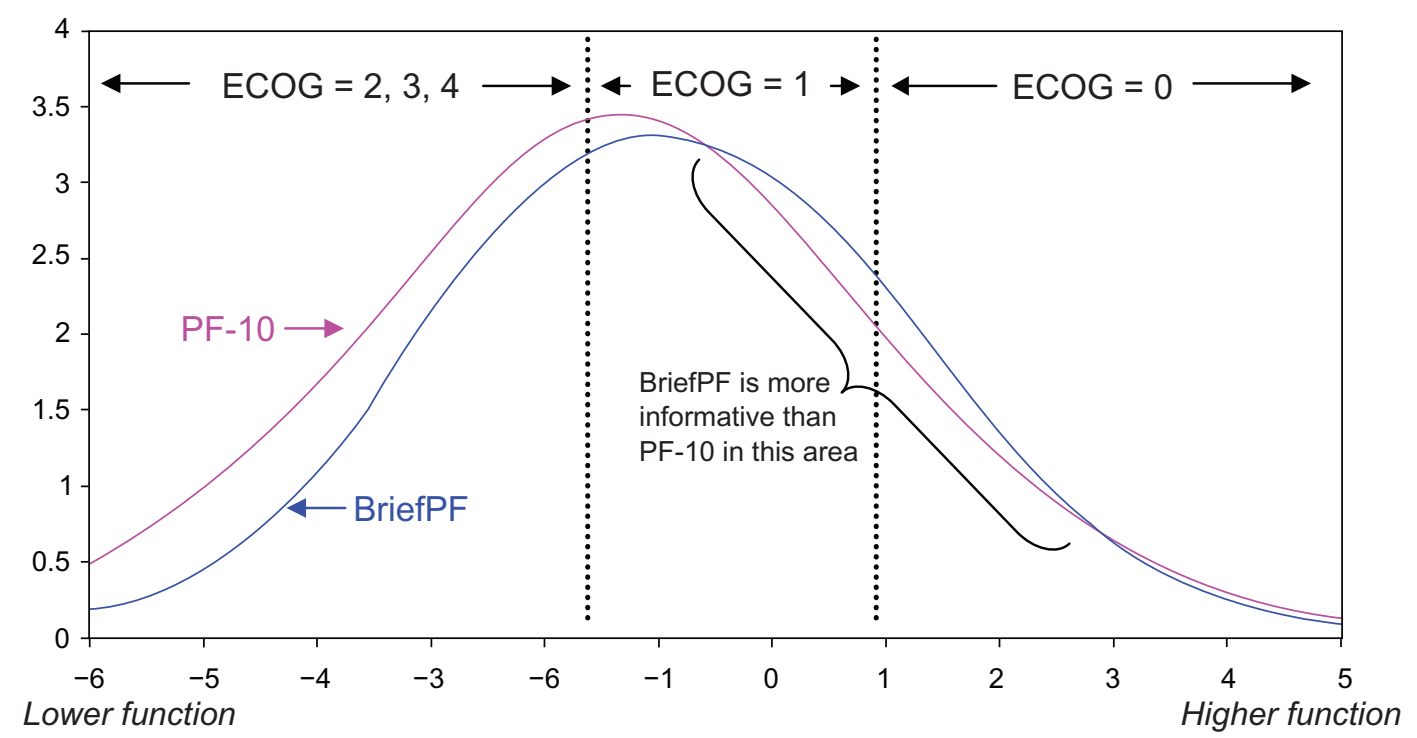

Figure I Comparison of scale information function curves of BriefPF and PF- 10 along the PF continuum. 
precision suggested the Rasch-based BriefPF outperformed both the PF-10 and the full PF item bank, we cautioned any further interpretation of relative precision, as precision in this context is merely a statement about the comparison of the ratio of explained and error variances between scales.

The observation that the BriefPF demonstrated higher information function for locating patients on the PF continuum among moderate to high functioning prostate cancer patients (according to ECOG PSR) is of particular interest. This may indicate that, compared to the PF-10, items in the BriefPF are more sensitive to activity limitations of men with prostate cancer. Since the clinical definition of disease and later stage prevalence has markedly shifted with the widespread use of PSA, the BriefPF may be particularly well suited to serve as an outcomes measure for the large proportion of men diagnosed and being treated for localized prostate cancer. ${ }^{30}$ Further study is required to more adequately determine whether the PF-10, which provides higher information function among the lower functioning patients, offers additional information beyond the BriefPF for men with more advanced disease.

While many prostate-specific HRQL instruments are available to measure physical function, the 5-item BriefPF is the shortest in length, without sacrificing precision or content validity. Its brevity may make it more appealing to alternatives, and may therefore improve the likelihood of routine assessment of PF in clinical practice, which is being recommended with increasing frequency in an era of accountability for, and comparative effectiveness of, available interventions. ${ }^{31}$ Merging the BriefPF with electronic data collection methods (eg, web testing) would further allow for more timely feedback that could support appropriate treatment decision-making. Recognizing its potential clinical utility, the National Institutes of Health Patient-Reported Outcomes Measurement Information System (NIH PROMIS, see: http:// www.nihpromis.org) has developed a PF item bank calibrated using the US general population, and the PROMIS PF item bank is further extended to the general cancer population via a PROMIS cancer supplement. Results of this study support the potential clinical and research contributions of the NIH PROMIS effort. Since there are common items shared by the PROMIS Cancer PF item bank and this current PF item bank, these two PF item banks can easily be referenced to one another on a common metric to standardize the measurement of PF across chronic diseases.

Several limitations of our study should be noted. First, our findings are based on a convenience sample of 128 patients. However, we recruited cancer patients from two very diverse geographic settings that include populations at risk for low health literacy skills and compromised cancer outcomes. Second, since validation of the BriefPF was based on cross-sectional data, we were unable to assess relationships between responses to the BriefPF and any particular health behavior or outcome. Future research should examine the predictive validity of the scale over time for treatment-related health services utilization and disease-specific outcomes. Finally, our scale might also benefit from further psychometric evaluation, including an investigation of test-retest reliability and discriminant validity. Evidence of sensitivity to change will be necessary to eventually determine whether the BriefPF is an applicable evaluative tool for various treatment strategies.

\section{Conclusion}

The BriefPF scale is a valid and precise measure for the practical and routine assessment of PF in clinical practice among patients with prostate cancer that improves upon the presently available pool of related HRQL instruments. Using itembanking methodology, our study reflects a strategy to simplify the measurement of PF and other HRQL domains through the application of a derived, tailored short form. The BriefPF allows for a more rapid, yet reliable, assessment of PF, and has considerable potential for widespread acceptance and use by physicians in the evaluation of patient-reported outcomes. This study reflects an ongoing effort to make evaluation tools meet the needs of clinicians by being easy to administer and time-efficient. Our hope is to achieve a common metric for HRQL domains, so as to improve how a patient's experience is processed in treatment decision-making, and gain the ability in the future to establish cross-comparisons of research studies that are unified in measurement.

\section{Acknowledgments}

The authors acknowledge grant support from the American Cancer Society (No. 01-221-01-PBP; PI: David Cella); National Cancer Institute (CA60068, PI: David Cella).

\section{References}

1. American Cancer Society. Cancer Facts and Figures 2009. Atlanta GA: America Cancer Society, 2009.

2. da Silva FC. Quality of life in prostatic cancer patients. Cancer. 1993; $72: 3803$.

3. Rossetti SR, Terrone C. Quality of life in prostate cancer patients. Eur Urol. 1996;30 Suppl 1:44.

4. Curran D, Fossa S, Aaronson N, et al; for European Organization for Research and Treatment of Cancer (EORTC), Genito-Urinary Tract Cancer Cooperative Group (GUT-CCG).Baseline quality of life of patients with advanced prostate cancer. Eur J Cancer. 1997;33: 1809-1814. 
5. Gralnek D, Wessells H, Cui H, et al. Differences in sexual function and quality of life after nerve sparing and nonnerve sparing radical retropubic prostatectomy. J Urol. 2000;163:1166-1170.

6. Potosky AL, Reeve BB, Clegg LX, et al. Quality of life following localized prostate cancer treated initially with androgen deprivation therapy or no therapy. J Natl Cancer. 2002;94(6):430-437.

7. Brandeis JM, Litwin MS, Burnison CM, et al. Quality of life outcomes after brachytherapy for early stage prostate cancer. J Urol. 2000; 163:851-857.

8. Osoba D, Tannock IF, Ernst DS, et al. Health-related quality of life in men with metastatic prostate cancer treated with prednisone alone or mitoxantrone and prednisone. J Clin Oncol. 1999;17:1654-1663.

9. Penson DF, Litwin MS, Aaronson NK. Health related quality of life in men with prostate cancer. J Urol. 2003;169:1653-1661.

10. Eton DT, Lepore SJ. Prostate cancer and health-related quality of life: a review of the literature. Psycho-oncology. 2002;11:307-326.

11. Eton DT, Lepore SJ, Helgeson VS. Early quality of life in patients with localized prostate carcinoma: an examination of treatment-related, demographic, and psychosocial factors. Cancer. 2001;92:1451-1459.

12. Litwin MS, Lubeck DP, Stoddard ML, et al. Quality of life before death for men with prostate cancer: results from the CaPSURE database. J Urol. 2001;165:871-875.

13. Penson DF, Stoddard ML, Pasta DJ, et al. The association between socioeconomic status, health insurance coverage, and quality of life in men with prostate cancer. J Clin Epidemiol. 2001;54:350-358.

14. Bylow K, Dale W, Mustian K, et al. Falls and physical performance deficits in older patients with prostate cancer undergoing androgen deprivation therapy. Urology. 2008;72(2):422-427.

15. Levy ME, Perera S, van Londen GJ, et al. Physical function changes in prostate cancer patients on androgen deprivation therapy: a 2-year prospective study. Urology. 2008;71:735-739.

16. Bode RK, Lai JS, Cella D, et al. Issues in the development of an item bank. Arch Phys Med Rehabil. 2003;84:S52-S60.

17. Bode RK, Lai JS, Dineen K, et al. Expansion of a physical function item bank and development of an abbreviated form for clinical research. J Appl Meas. 2006; 7:(1):1-15.

18. Sloan JA, Loprinzi CL, Kuross SA, et al. Randomized comparison of four tools measuring overall quality of life in patients with advanced cancer. J Clin Oncol. 1998;16:3662-3673.
19. McHorney CA, Ware JE Jr, Raczek AE. The MOS 36-Item Short-Form Health Survey (SF-36): II. Psychometric and clinical tests of validity in measuring physical and mental health constructs. Med Care. 1993; 31:247-263.

20. Bode RK, Cella D, Lai JS, et al. Developing an initial physical function item bank from existing sources. J Appl Meas. 2003;4(2): 124-136.

21. Schag CA, Ganz PA, Heinrich RL. Cancer Rehabilitation Evaluation System - short form (CARES-SF). A cancer specific rehabilitation and quality of life instrument. Cancer. 1991;68:1406-1413.

22. Cella DF, Tulsky DS, Gray G, et al. The Functional Assessment of Cancer Therapy scale: development and validation of the general measure. J Clin Oncol. 1993;11:(3)570-579.

23. Aaronson NK, Ahmedzai S, Bergman B, et al. The European Organization for Research and Treatment of Cancer QLQ-C30: a quality-of-life instrument for use in international clinical trials in oncology. $\mathrm{J} \mathrm{Natl}$ Cancer Inst. 1993;85:(5)365-376.

24. Spitzer WO, Dobson AJ, Hall J, et al. Measuring the quality of life of cancer patients: a concise QL-index for use by physicians. J Chronic Dis. 1981;34:585-597.

25. Ware JE Jr, Sherbourne CD. The MOS 36-item short-form health survey (SF-36). I. Conceptual framework and item selection. Med Care. 1992; 30:473-483.

26. Tarlov AR, Ware JJE, Wells KB. Medical Outcomes Study, 1986-1992 (Data Set 30-34, Umayahara M and Lang EL). Boston, MA: The Health Institute, New England Medical Center.

27. Oken MM, Creech RH, Tormey DC, et al. Toxicity and response criteria of the Eastern Cooperative Oncology Group. Am J Clin Oncol. 1982; 5:649-655.

28. McHorney CA, Ware JE Jr, Rogers W, et al. The validity and relative precision of MOS short- and long-form health status scales and Dartmouth COOP charts. Results from the Medical Outcomes Study. Med Care. 1992;30:MS253-MS265.

29. Linacre UM. From microscale to winsteps: 20 years of Rasch Software development. Rasch Measurement Transactions. 2004;17(4):958.

30. Cooperberg MR, Lubeck DP, Mehta SS, et al. Time trends in clinical risk stratification for prostate cancer: implications for outcomes (data from CaPSURE). J Urol. 2003;170:S21-S27.

31. Jacobsen PB, Davis K, Cella D. Assessing quality of life in research and clinical practice. Oncology. 2002;(Williston Park),16:133-139.
Patient Related Outcome Measures

\section{Publish your work in this journal}

Patient Related Outcome Measures is an international, peer-reviewed, open access journal focusing on treatment outcomes specifically relevant to patients. All aspects of patient care are addressed within the journal and practitioners from all disciplines are invited to submit their work as well as healthcare researchers and patient support groups. Areas covered will

\section{Dovepress}

include: Quality of life scores; Patient satisfaction audits; Treatment outcomes that focus on the patient; Research into improving patient outcomes; Hypotheses of interventions to improve outcomes; Short communications that illustrate improved outcomes; Case reports or series that show an improved patient experience; Patient journey descriptions or research. 